\title{
Qualitative Examination of Credit Based Shaper in IEEE 802.1AVB
}

\author{
LokaManjari. G, Nikhila. K, K.. Annapurna, B. Seetha Ramanjaneyulu
}

\begin{abstract}
Switched Ethernethasenteredinto industrial networking applications more than a decade ago. But the concern of non-determinism about its transmissions is still to be addressed. It is the main issue in industrial applications where the transmissions need to be time-sensitive. AVB (Audio Video Bridging) is one mechanism that can help in improving this determinism. AVB is a set of technical standards used to transport the low-latency streaming services over Ethernet networks. In order to provide low latency, AVB specify the traffic shaping mechanisms that are used to serialize the set of frames. Traffic shaping and transmission selection can be performed by the Credit Based Shaper in AVB. In this paper we analyze the credit based shaper by changing the payload of $A V B$ and observing the impact on best effort traffic. Analysis results indicate that it offers good throughputs at appropriate load conditions.
\end{abstract}

Key words: Industrial Ethernet, Time sensitive networking, Deterministic Ethernet, Credit based shaper, Audio-Video bridging.

\section{INTRODUCTION}

Ethernet is used as a universal network solution in many industrial applications. Now, industrial environment demands for real time communication. To provide real timeenvironment,various automation vendors developed their own protocols like Ether IP, profibus, Ether CATetc. Inallthesetechnologiesadditionaladaptationshave

to be made to meet the real time requirements [1]. To fulfill this real timeobjective, new standard was proposed by IEEE 802.1 working group i.e. Audio Video Bridging (AVB). The IEEE AVB is a set of standards focusing on transporting low latency streaming services.

IEEE 802.1 AVB task group is working on standards to meet the requirements [2]. IEEE 802.AS Precision Time Protocol is key requirement in real time networked application domains. It is possible to synchronize in the sub micro second range[3].IEEE 802.Qat isStream Reservation Protocol (SRP). This standard describes how stream reservation may be established between talker and listener in a bridge.IEEE 802.1Qav is about forwarding and queuing enhancements. It shapes the network traffic so that latency and bandwidth of reserved streams can be controlled.

Revised Manuscript Received on December 16, 2019

LokaManjari. G, Department of ECE, VFSTR University, Vadlamudi, Guntur, AP, India

gaddelokamanjari14@gmail.com

Nikhila. K, Department of ECE, VFSTR University, Vadlamudi, Guntur, AP, India

karinikhila@gmail.com

K. Annapurna, Department of ECE, VFSTR University, Vadlamudi, Guntur, AP, India

arya.anu85@gmail.com

B. Seetha Ramanjaneyulu, Department of ECE, VFSTR University, Vadlamudi, Guntur, AP, India

ramanbs@gmail.com

\section{BACKGROUND AND RELATEDWORK}

Overview of Audio video Bridging: $A V B$ is a set of technical standards developed by the institute of electrical and electronics engineering (IEEE). AVB provides the specifications that will allow time synchronized low latency streaming services through IEEE802 networks. AVB consists of the following:

\section{IEEE 802.1AS Precision Time Protocol}

It is used to synchronize the local clocks in the network for less than 1milli second .Synchronization can be done by using two types of clocks.

A master clock that can be controlled by GPS receiver synchronizes the respective slaves connected to it[3].

IEEE 802.1Qat Stream Reservation Protocol (SRP)

SRP is used to reserve the bandwidth dynamically. The bandwidth reservation can be done in two classes, 'Class A' and 'class B'. Classification can be done based on the priority code point (PCP) in the VLAN tag. 'Class $A$ ' is having a latency of about $2 \mathrm{~ms}$ over 7 hops. 'Class B' is having a latency of about 50ms over 5hops [5].

The node that wants to reserve the bandwidth will send talker advertise attribute to the connecting devices. This attribute consists of the following.Stream ID represents the MAC address of the talker.Data frame parameters specifies the destination MAC address. Traffic specification defines the Maximum Frame Size and Maximum Interval Frames. Maximum Frame Size means how much data we are sending without overhead (for example 350 bytes).Maximum Interval Frames means the number of frames the talker may send within a Class Measurement Interval(CMI). Depending upon traffic priority class, CMI is given. For 'class A' CMI is 125 micro seconds and for 'class B'it is 250 micro seconds [6].

The talker advertisestheattribute message which is received by therecipient.Itwill register first and then it will calculate the bandwidth available. The formula for calculating the requiredbandwidth is [7],

\footnotetext{
B.W $=\frac{\text { TspecMlaxInterwalFrames }}{\text { CMI }} \times 8 \times($ TspecMaxFS + overhead $)$

Where FS=Frame Size
}

If the bandwidth is available, the message will be forwarded to the neighboring devices until it reaches the destination. If the listener receives the 'talker advertise' message, then it will send 'listener ready' attribute until it reaches the talker, if it is interested in the advertised stream.

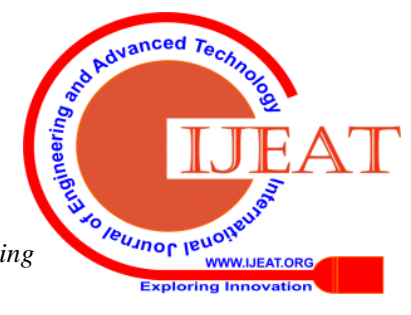




\section{Qualitative Examination of Credit Based Shaper in IEEE 802.1AVB}

AVB traffic can reserve the bandwidth maximum up to $75 \%$ and the remaining $25 \%$ bandwidth is allocated to Best Effort traffic [8]. The bandwidth which is not used by AVB traffic will be allocated to the Best effort (BE) traffic

Transmission selection and traffic shaping mechanisms are carried out here. Credit Based Shaper (CBS) is one of the earliest traffic shapers standardized in AVB.This shaper allows two types of traffic. One is AVB trafficandanother is Best effort traffic. AVB traffic is associated with audio and video applications that are required to be delivered within bounded latency. AVB traffic is handled bycredit based shaper as shown in Figure-1. The AVB traffic is having highest priority than best effort traffic [9]. In BE trafficthere is no timing guarantee in the delivery of packets. $\mathrm{BE}$ traffic is given to strict priority algorithm.

In CBS, the transmission of a frame is allowed when the credits are available for the associated class.

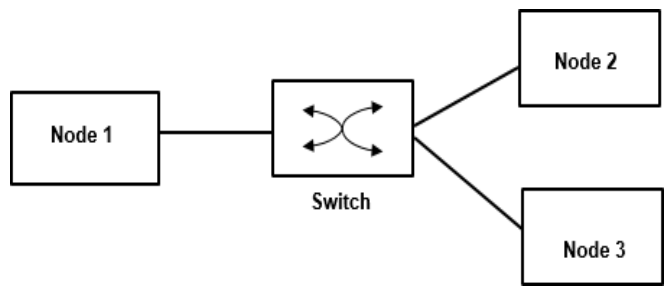

Figure-1 IEEE 802.1 Qav forwarding and Queuing Enhancements

CBS defines the Class Measurement Interval. Within each CMI, each AVB talker may send up to defined limitset by the SRP.AVB talker may send one Ethernet frame as per configurable CMI. Credit Based shaper has the following rules[10]. The transmission of AVB frame will be allowed when the credit iszeroor positive. When AVB frame is being transmitted, the credit starts decreasing with a rate called'send slope'. When AVB frame is queued, but not transmitted, the credit starts increasing with a rate called'idle slope'. It is shown in Figure-2.

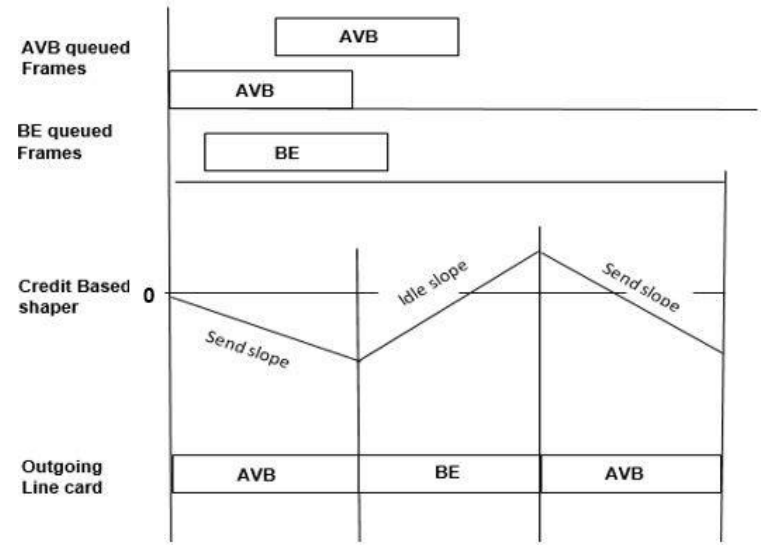

Figure-2 Operation of the Credit Based Shaper algorithm

\section{IEEE 802.1Qav Forwarding and Queuing Enhancements}

\section{EXPERIMENTS AND RESULT DESCRIPTION}

A model of the network is generated and analyzedusing CoRE4INET [11]. It is an extension to the INET-Framework for the event based simulation of real time Ethernet in the OMNeT++ simulation system [12]. Simulation time was set to $5 \mathrm{~ms}$. Bandwidth was set up to $100 \mathrm{Mbit} / \mathrm{s}$ with fullduplex.

Models of different networksare designed and analyzed to find out the performance ofcredit based shaper by changing the payload of $\mathrm{AVB}$ and observing the impact on BE traffic. Transmission period of AVB traffic was set based on the AVB standard and BE traffic was set to 50us. Payload was set as 1500 Byte.

A. Impact on Best Efforttraffic when changing the 'class A' payload when 'class B'isconsidered

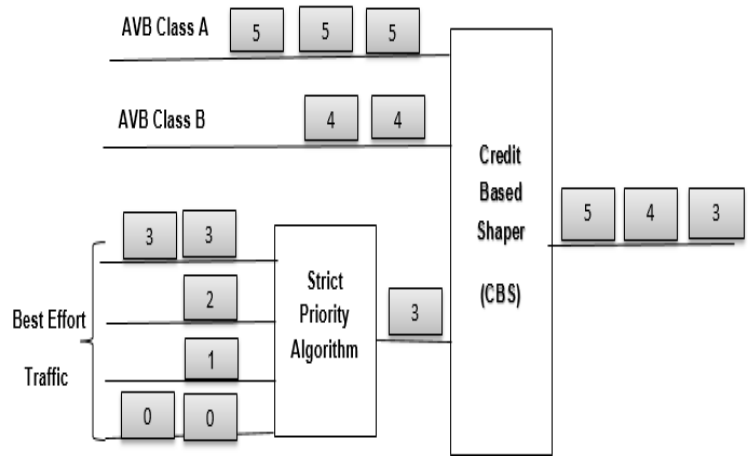

Figure-3 Network Topology 1

In Figure-3, Node 1 sends 'class A' traffic and BE traffic to Node3.Node2 sends 'class B' traffic to Node3. These nodes are interconnected by a Switch.Herepayload of 'class A' is taken as 350 Bytes andstarts

Increasing. Its impact on best effort traffic is observed. It is shown in Figure-4.

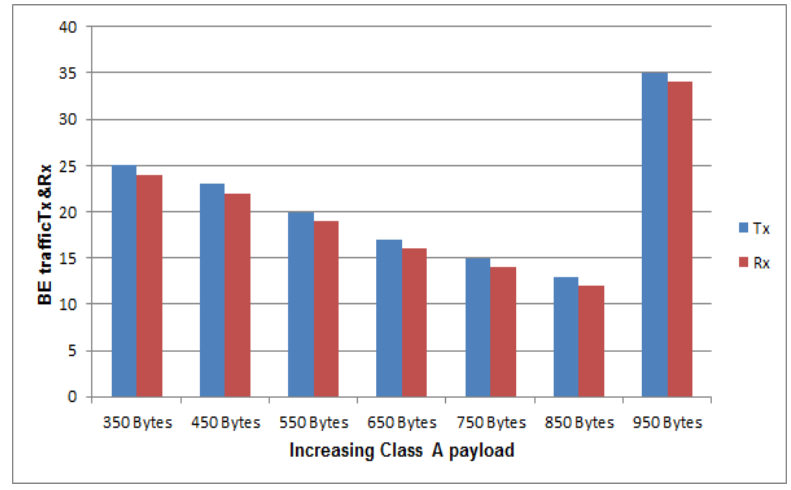

Figure-4 Impact on Best Effort traffic when changing the 'class A' payload when 'class $B$ ' is considered

When 'class A' payload increases, then number of BE frames being transmitted and received decreases, because in SRP protocol the bandwidth allocated for AVB is $75 \%$ and for BE traffic it is $25 \%$. So if the AVB traffic does

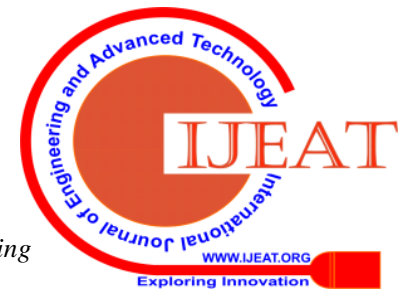


not use full $75 \%$, thenthat is allocated to the BE traffic. When we increase the AVB 'class A'payload,then more bandwidth will be occupied by AVB traffic and less band width is left to Best Efforttraffic.

However, for the case of 950 bytesAVBframe payload, the best transmitted and received frames of best effort traffic have increased. It is due to the fact that that 950 Bytes frame of AVB 'class A'is beyond the maximum permitted bandwidth of $75 \%$.

B.W of class A

$=1 / 125$ us $\times 8 \times(950$ bytes +50 Bytes $)$

$=64 \mathrm{Mbit} / \mathrm{s}$

Here we are considering AVB 'class B' traffic also. Payload of 'Class B'is considered as 350Bytes. If we calculate Bandwidth for 'class B', it is:

B.W for 'class B' $=1 / 250$ us $\times 8 \times($ TspecMaxFrameSize

+overhead)

$=1 / 250$ us $\times 8 \times(350$ Bytes +50 Bytes $)$

$=12.8 \mathrm{Mbit} / \mathrm{s}$

If we add the bandwidths of both 'class A'and'class B', then

Required B.W=64Mbit/s+12.8Mbit/s

$=76.8 \mathrm{Mbit} / \mathrm{s}$

It is crossing the reserved B.W 75\%. So 'class A' traffic won't be sent. This Bandwidth from Node 1 will be consumed by BE Traffic. So, for this 950 byte frame of 'Class A', highest BE throughput occurs.

B. Impact on Best Effort Traffic when 'class A' payload changes when 'class $B$ ' is notconsidered

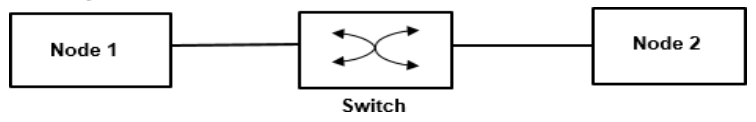

Figure5. Network Topology 2

In Network 2, Node 1 is sending 'class A' traffic and as well as BE traffic to Node 2. Here 'class B' traffic is not considered. 'Class A' payload is taken as 350Bytes and starts increasing.Impactof this increase on $\mathrm{BE}$ traffic is shown inFigure-6

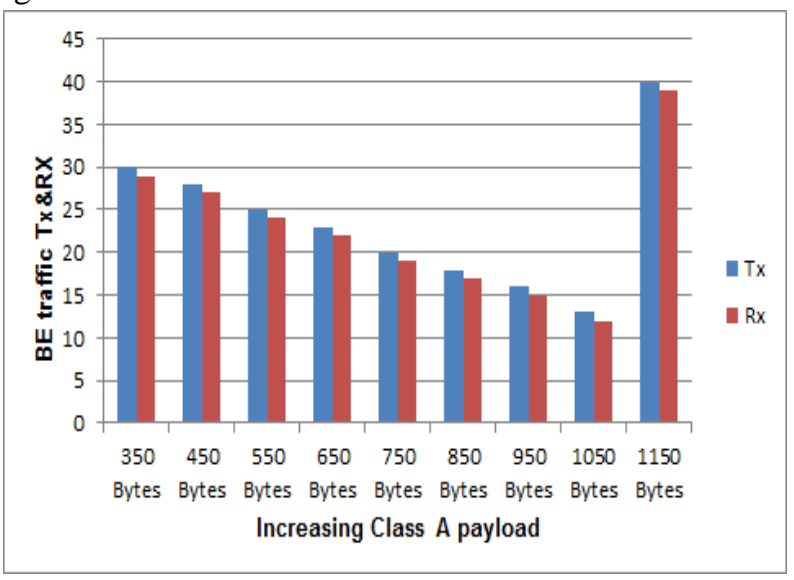

Figure-6 Impact on BE traffic when 'class A' payload changes when 'class $B$ ' is notconsidered
Here, 'class B' is not considered. The bandwidth reserved for AVB will be occupied entirely by class A only. Depending on the payload of class A, how much band width the 'class A' wants will be decided andused. Remaining bandwidth will be given to BE traffic. So, Node 1 here transmits more BE traffic when compared to Figure-4. Similar toprevious scenario, here alsoBE traffic decreases when AVB 'class A' payload increases. The reason is same as in the previous scenario. The difference between Figure- 4 and Figure- 6 is that, 'class $A$ ' is able to send up to 1050 Bytes compared to 850 Bytes earlier. This is due to the absence of 'class B' frames.

For the case of 1150Bytes payload from 'class A', it won't send because the traffic because it is crossing the reserved bandwidth limit of $75 \%$. These Bandwidth calculation is given below:

B.W for class $A=\frac{1}{125 u s} \times 8 \times(1150+50)$

$=76.8 \mathrm{MBps}$

So Node 1stops transmitting 'Class A', for the cases of 1150 bytes and beyond. Then total bandwidth will be occupied by BE traffic in Node 1. So higher volumes of BE type traffic is being sent and received.

C. Impact on Best Effort Traffic when 'class B' payload changes when 'class A'isconsidered

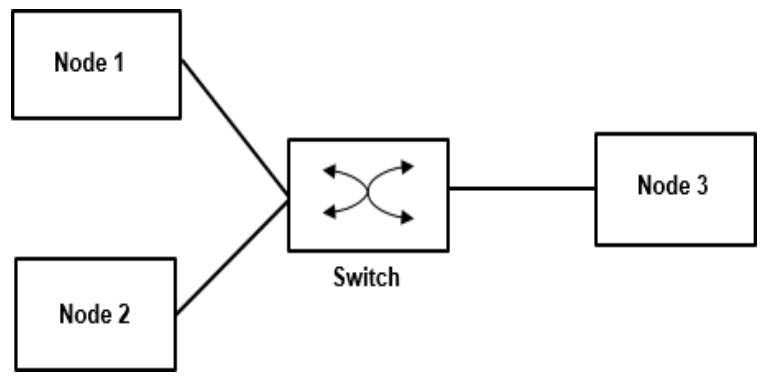

Figure-7 Network Topology3

In Network 3, Node 1 is sending 'class A' traffic to Node 3. Node 2 is sending 'class $B$ ' and BE traffic to Node3. The reserved bandwidth for AVB will be occupied by 'class $A$ ' and 'class B' here. Observations are made in this network by increasing 'classB'payload .Impact of it on BE traffic is observed and results are given in Figure-8. 


\section{Qualitative Examination of Credit Based Shaper in IEEE 802.1AVB}

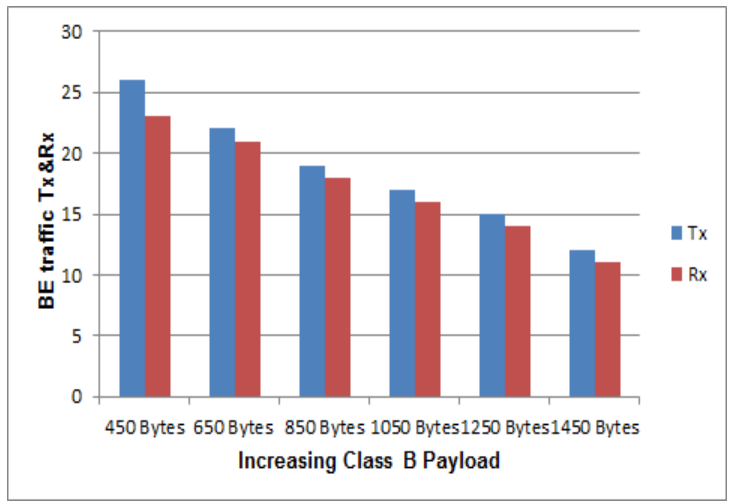

Figure-8 Impact on Best Effort Traffic when class B payload changes when class $A$ is considered

Here 'class $A$ ' is considered as 350 Bytes andincreasing payloads of up to 1450 Bytes are considered for 'class B'. Bandwidth calculation is given below:

B.W for class $\mathrm{A}=\frac{1}{125 \mathrm{us}} \times 8 \times(1150+50)$

$\frac{1}{125 u s} \times 8 \times(350+50)$

$=25.6 \mathrm{MBps}$

B.W for classB $=\frac{1}{250 \text { us }} \times 8 \times(1450+50)$

$=48 \mathrm{MBps}$

If we add both 'class A'\&'class B'B.W=25.6MBps+48MBps $=73.6 \mathrm{MBps} . . .(5)$

In 'class B',wecan't increase more than 1500 bytes because the Ethernet's maximum frame size of 1500Bytes is chosen.

D. Impact on Best Effort Traffic when changing the 'class B' payload when 'class A' is notconsidered

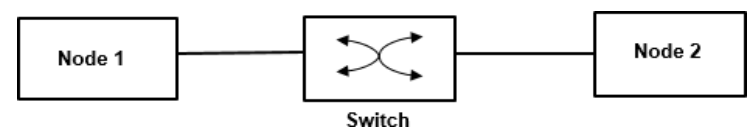

Figure-9 Network Topology 4

In Network 4, Node 1 sends 'class B' traffic and BE traffic to Node 2. Here 'class B' payload increases and the impact of it on BE traffic is observed.Results are shown in figure 10.

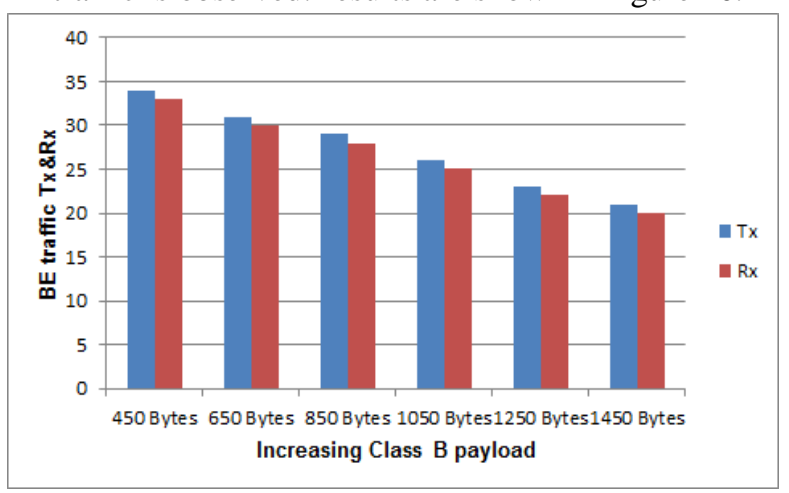

Figure-10 Impact on BE Traffic when changing the 'class $B$ ' payload when 'class $A$ ' is notconsidered

'In this network 'class A' is not considered so the reserved bandwidth for AVB will be occupied by 'class B' only. 'Class B' will occupy less bandwidth when compared to 'class A' due to CMI. So BE traffic is sending more packets when compared to figure8.

Here 'Class B' is sending up to 1450 Bytes .It is due to the available bandwidth. Calculation of bandwidth for 'class B' is given below.

$$
\begin{aligned}
\text { B.W for 'class B' } & =\frac{1}{250 u s} \times 8 \times(1450+50) \\
& =48 \mathrm{MBps} \ldots(6)
\end{aligned}
$$

\section{CONCLUSION}

In this paper an analysis of credit based shaper is carried out forvarying load conditions and network scenarios. Credit Based Shaper (CBS) algorithm is used to shape the transmission of stream in accordance with the bandwidth that was reserved on a given outbound queue. Various situations of changing the payload of AVB and observing the impact on Best effort traffic are carried out. Results indicate that choosing appropriate allocations depending on the load conditions will help to maximize the throughputs.

\section{REFERENCES}

1. The Future of Industrial Communication [online].Avilable:https://www.hsowl.de/init/uploads/tx_initdb/IEEEMa gazine.pdf

2. Michael D. JohasTeener, Andre N. Fredette, Christian Boiger, Philippe Klein, Craig Gunther, David Olsen, and Kevin Stanton"Heterogeneous Networks for Audio and Video: Using IEEE 802.1 Audio Video Bridging" 2013 IEEE

3. White Paper Precision Clock Synchronization [online].Available:www.industrialnetworking.com/pdf/Hirsch mann_IEEE_1588.pdf

4. Martin L'evesque and David Tipper " $p t p++$ : A Precision Time Protocol Simulation Model for OMNeT++ / INET "Proceedings of the "OMNeT++ Community Summit 2015.

5. Philipp Meyer, Till Steinbach, Franz Korf, and Thomas C. Schmidt, "Extending IEEE 802.1 AVB with Time-triggered Scheduling: A Simulation Study of the Coexistence of Synchronous and Asynchronous Traffic," IEEE Vehicular Networking Conference (VNC), pp. 47-54, December 2013.

6. Levi PearsonAVnu Alliance Best Practices Stream Reservation Protocol [online]. Available:avnu.org/wp content/uploads/2014/05/AVnu_Stream-Reservation-Protocol-v1.pdf

7. Industrial Communication Technology Handbook, Second Edition edited by Richard Zurawski [online]. Available: https://books.google.co.in/books?isbn=148220732X

8. Mohammad Ashjaei, Gaetano Patti, Moris Behnam, Thomas Nolte, GiulianaAlderisi, Lucia Lo Bello, "Schedulability analysis of Ethernet Audio Video Bridging networks with scheduled traffic support",springer ,February 2017

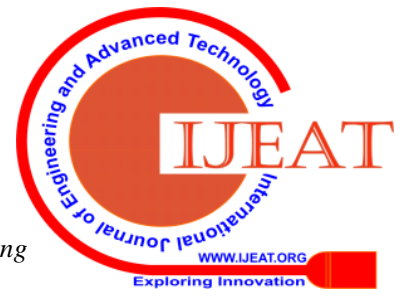


9. T. Steinbach, H.-T. Lim, F. Korf, T. C. Schmidt,D.Herrscher, and A. Wolisz, "Tomorrow's In-Car Interconnect? A Competitive Evaluation of IEEE802.1 AVB and Time-Triggered Ethernet (AS6802)," in 2012 IEEE Vehicular Technology Conference (VTC Fall). Piscataway, New Jersey: IEEE Press, Sep. 2012.

10. IEEE Std. 802.1Qav, IEEE Standard for Local and metropolitan area networks, Virtual Bridged Local AreaNetworks, Amendment 12: Forwarding and Queuing Enhancements for Time-Sensitive Streams, January 2010

11. Communication over Realtime Ethernet for INET Framework (CoRE4INET) [online]: https://core4inet.core-rg.de

12. OMNeT++ Community, "OMNeT++ 5.1.1" [Online]: http://www.omnetpp.org 